\section{Fatores associados à hipertensão arterial sistêmica em Nobres-MT}

\author{
Factors associated to systemic \\ arterial hypertension in Nobres-MT
}

\section{Resumo}

Objetivo: Identificar fatores associados à Hipertensão Arterial Sistêmica (HAS) no município de Nobres-MT. Método: Foi realizado um estudo de corte transversal, em Nobres-MT, de janeiro a março de 2006, em 1.003 indivíduos com idade superior a 18 anos, residentes na zona urbana do município. Os dados foram coletados por entrevista domiciliar, sendo aferidos peso, estatura, circunferência da cintura e pressão arterial (PA). Foram considerados hipertensos aqueles com PA $\geq 140 / 90$ mmHg. Para identificar controle dos fatores de confundimento aplicou-se o modelo de regressão de Poisson, com estimativas de razão de prevalências ajustadas e seus respectivos intervalo de confiança de 95\%. Resultados: Foram avaliados 1.003 indivíduos, sendo 51,3\% do gênero masculino. A prevalência de HAS foi $30,1 \%$, maior no sexo feminino $(31,1 \%)$ do que no sexo masculino (29,1\%). A análise ajustada confirmou a associação independente entre HA e o avançar da idade e com Índice de Massa Corporal igual ou superior a $25 \mathrm{~kg} /$ $\mathrm{m}^{2}$. Conclusão: Reafirma-se a necessidade de ações em nível populacional e individual direcionadas ao controle de peso corporal, principalmente em indivíduos com idade superior a 30 anos, que possivelmente impactariam de forma importante para a prevalência de HAS.

Palavras-chave: Hipertensão. Epidemiologia. Fatores de risco cardiovascular.

Tânia Maria do Rosário'

Luiz Cesar Nazário Scala'

Giovanny Vinícius Araújo de França'

Márcia Regina Pereira'

Paulo Cesar Brandão Veiga Jardim"

'Instituto de Saúde Coletiva da Universidade Federal de Mato Grosso

" Faculdade de Medicina da Universidade Federal de Goiás 


\section{Abstract}

Objective: To identify the factors associated to systemic arterial hypertension (SAH) in Nobres -MT, Brazil. Method: A cross-sectional study was carried out in Nobres-MT, from January to March, 2006; 1003 individuals aged more than 18 years, resident in the urban region were analyzed. Data was obtained through household questionnaires. Measurements of weight, height, waist circumference and blood pressures (BP) were taken. The second of three BP measurements was used in the analysis; $\mathrm{SAH}$ was defined as $\mathrm{BP} \geq 140 / 90 \mathrm{mmHg}$. The Poisson regression method was used for controlling confounding factors, with adjusted prevalence ratio (PR) estimates and their respective $95 \%$ confidence intervals. Results: 1003 individuals were evaluated (51.3\% male). SAH prevalence was $30.1 \%$, and it was higher among women than men ( $30.1 \%$ vs. $20.9 \%$ ). Adjusted analysis confirmed independent association between SAH, aging and body mass index equal or over 25 $\mathrm{Kg} / \mathrm{m} 2$. Conclusion:We reaffirm the need of actions both at population and individual levels targeting weight control, especially for people over 30 years of age, which could significantly reflect on SAH control.

Keywords: Hypertension; Epidemiology; Cardiovascular risk factors.

\section{Introdução}

As doenças cardiovasculares (DCV) são as principais causas de morbidade e mortalidade em todo mundo. No ano 2000, essas doenças contribuíram para $62,9 \%$ da mortalidade nas Américas ${ }^{1}$.

Entre as DCV, o acidente vascular encefálico (AVE), ocupa o primeiro lugar entre as causas de morte no Brasil ${ }^{2}$. A hipertensão arterial é o fator de risco mais importante para ocorrência dos AVEs, tanto isquêmicos como hemorrágicos ${ }^{3}$. Neste contexto, a hipertensão arterial sistêmica, devido ao seu importante papel como fator de risco cardiovascular modificável ${ }^{4,5}$, revela-se como um dos mais relevantes problemas de saúde pública do país, com prevalência entre $22,3 \%$ a $43,9 \%$, dependendo da população estudada, da região ou do critério diagnostico utilizado ${ }^{6}$.

Devido à magnitude e importância da doença no Brasil, e a escassez de dados relativos à sua prevalência na região CentroOeste, especialmente em Mato Grosso e Goiás, a Universidade Federal de Goiás (Faculdade de Medicina e Liga de Hipertensão Arterial da Universidade Federal de Goiás), em conjunto com a Universidade Federal de Mato Grosso (Instituto de Saúde Coletiva e Faculdade de Ciências Médicas), realizaram estudo multicêntrico, com o objetivo de determinar a prevalência e as características epidemiológicas da hipertensão arterial na região Centro-Oeste, especificamente nas cidades de Goiânia e Firminópolis (Goiás) e Cuiabá e Nobres (Mato Grosso). Este estudo objetivou identificar fatores associados à Hipertensão Arterial Sistêmica no município de Nobres-MT.

\section{Método}

Estudo transversal, de base populacional, realizado entre 18 de janeiro 29 de março de 2006 em Nobres, município localizado na região norte do Estado de Mato Grosso, com população total estimada de 12.269 habitantes ${ }^{7}$. A população-alvo avaliada consistiu de indivíduos de ambos os sexos, 
com idade entre 18 a 90 anos, residentes na área urbana do município.

Para determinação do tamanho da amostra foram considerados os seguintes parâmetros: nível de significância de 5\% (bicaudal), erro fixado em $2,5 \%$ (d), poder de $80 \%$ supondo-se uma prevalência de hipertensão arterial entre adultos de $20 \%^{8}$, contemplando-se $9 \%$ para controle de fatores de confusão e a inexistência de perdas e recusas devido à possibilidade de reposição. Deste modo, a amostra mínima consistiu de 1.003 indivíduos.

O delineamento da amostra foi realizado em quatro estágios. Primeiramente, foram incluídos todos os 13 setores censitários da zona urbana de Nobres-MT, procedendose ao sorteio de quadras contidas em tais setores. Dos 3.619 domicílios presentes nas quadras sorteadas, 1.003 foram sorteados. Após identificação dos mesmos, sorteou-se um único indivíduo em cada um deles, de idade igual ou superior a 18 anos, que foi convidado a participar do estudo. Aqueles que não aceitaram participar foram substituídos por residentes em casas contíguas à direita.

A equipe de pesquisa foi constituída pelo coordenador geral do projeto em Mato Grosso, pela coordenadora e supervisora de Nobres, autora deste estudo, e pelos entrevistadores, em sua maioria agentes comunitários de saúde do município. A supervisora foi responsável por acompanhar continuamente o trabalho de campo, participando de reuniões semanais para avaliação do desempenho e das tarefas realizadas, e seis duplas de entrevistadores de campo. Os entrevistadores foram devidamente treinados e orientados para realização do trabalho de campo. Os mesmos eram responsáveis pela abordagem no domicílio, estando devidamente identificados por meio de crachás, camisetas e bolsas com a logomarca da pesquisa.

Os dados foram obtidos por meio de aferição da pressão arterial (PA), aplicação de questionário estruturado, e mensurações antropométricas. Utilizou-se, para aferição da PA, o aparelho semi-automático da marca OMROM-HEM $705 \mathrm{CP}^{9}$, validado internacionalmente, estando os indivíduos sentados, com braço esquerdo apoiado sobre a mesa à altura do coração, palma voltada para cima, certificando-se de que o mesmo não estivesse com a bexiga cheia, não houvesse praticado exercício físico ou ter fumado ou ingerido bebida alcoólica nos trinta minutos antecedentes às medidas. Utilizou-se braçadeira compatível com a circunferência do braço.

Foram realizadas duas aferições da PA, no início e término da entrevista. Para fins de análise foi considerada a última, desde que não houvesse diferença superior a $5 \mathrm{mmHg}$ entre as mesmas. Em caso contrário, eram realizadas mais medidas complementares, com intervalo mínimo de três minutos, até que as diferenças entre as mesmas fosse igual ou inferior a $5 \mathrm{mmHg}$, ou até completar cinco medidas, e o valor final da PA registrado, nesse caso foi a média das duas últimas aferições.

Para definição do desfecho, foram considerados hipertensos os indivíduos que apresentaram pressão arterial sistólica superior a 140mmHg e/ou pressão arterial diastólica superior a $90 \mathrm{mmHg}$, com base nas V Diretrizes Brasileiras de Hipertensão Arterial ${ }^{6}$.

Aplicou-se um questionário estruturado, padronizado e testado em um estudo piloto, que era respondido pelo morador sorteado. Foram analisados os seguintes aspectos socioeconômicos e demográficos: idade, expressa em anos completos e categorizada em faixas etárias de 18 a 29 anos, 30 a 39 anos, 40 a 49 anos, 50 a 59 anos, 60 a 69 anos, 70 anos ou mais; gênero, masculino e feminino; situação conjugal, se os entrevistados viviam com ou sem companheiro (a) na época do estudo; escolaridade, categorizada em anos de estudo $<8, \geq 8$ ou não ter freqüentado a escola; renda familiar mensal per capita, segundo valores de salários mínimos $(\mathrm{R} \$ 300,00)$.

Avaliou-se, ainda, questões comportamentais como tabagismo, consumo de bebidas alcoólicas e atividade física, tendo com base questões padronizadas baseadas no 
protocolo do estudo CARMEN (Conjunto de Ações para a Redução Multifatorial de Enfermidades Não Transmissíveis) ${ }^{10}$. Com relação ao tabagismo, os participantes foram categorizados em três grupos: os que nunca fumaram; os ex-fumantes, ou seja, aqueles que pararam de fumar há pelo menos seis meses, anteriores à realização do estudo; $\mathrm{e}$ os fumantes, sendo estes indivíduos que se autodeclararam fumantes no momento da entrevista, independente da quantidade de cigarros consumida por dia.

Foram considerados consumidores de bebidas alcoólicas todos aqueles que referiram fazer uso delas, independentemente do tipo ou quantidade. A prática de atividade física foi considerada em quatro categorias: ativo, irregularmente ativo A, irregularmente ativo B e sedentário, segundo consenso realizado entre o CELAFISCS e o Center for Disease Control (CDC) em $2002^{11}$. Questionou-se ainda, sobre o hábito de consumir regularmente, nos seis meses precedentes ao estudo, alimentação rica em gordura, café, refrigerante, guaraná em pó e adição de sal à mesa, sendo todas variáveis dicotômicas (sim/não).

A massa corporal e a estatura foram aferidos segundo técnica padronizada de $\mathrm{LOH}$ MAN et at ${ }^{12}$, utilizando-se balanças digitais da marca Plenna, com capacidade para $150 \mathrm{Kg}$ e precisão de $200 \mathrm{~g}$, e estadiômetros da marca SECA, respectivamente. As circunferências da cintura e do quadril foram aferidas segundo técnica de Callaway et $\mathrm{al}^{13}$, utilizando-se fitas métricas flexíveis e inextensíveis, da marca Cardiomed. Para análise, calculou-se o Índice de Massa Corporal $\left(\mathrm{IMC}=\right.$ peso $/$ estatura $\left.^{2}\right)$, categorizando os indivíduos em 4 grupos: inferior a $18,5 \mathrm{Kg} / \mathrm{m}^{2}$, de 18,5 a $24,9 \mathrm{Kg} / \mathrm{m}^{2}$, de 25,0 a $29,9 \mathrm{Kg} / \mathrm{m}^{2} \mathrm{e}$ igual ou superior a $30 \mathrm{Kg} / \mathrm{m}^{2}$, para definição, respectivamente, de baixo peso, eutrofia, sobrepeso e obesidade. A circunferência da cintura foi considerada aumentada quando a medida foi igual ou superior a $94 \mathrm{~cm}$ para homens e igual ou superior a $80 \mathrm{~cm}$ para mulheres, sendo classificada como muito aumentada caso fosse igual ou superior a $102 \mathrm{~cm} \mathrm{e} 88 \mathrm{~cm}$ para homens e mulheres, respectivamente. Foram considerados em risco de doença cardiovascular os homens com razão cintura-quadril superior a 1 e as mulheres com razão superior a $0,85^{14}$.

O controle de qualidade da coleta de dados constituiu no acompanhamento e verificação das informações coletadas nos domicílios. Os questionários em branco eram entregues diariamente aos entrevistadores, recolhendo-se os já preenchidos, que, por sua vez, eram revisados pelo coordenador local. Cerca de $10 \%$ dos domicílios sorteados foram novamente visitados ou contatados por telefone pela coordenadora. Os dados foram digitados em duplicata no programa Epi Info 2000, utilizando-se o recurso Data Compare do mesmo para correção das diferenças entre os bancos de dados. Foram verificadas, ainda, possíveis inconsistências, sendo as mesmas corrigidas.

Para análise de dados foram utilizados os programas Epi Info $2000^{15}$ versão 3.3.2 e Stata ${ }^{16}$ versão 10.0. Nas análises foi controlado o efeito do delineamento, presente por se tratar de um estudo amostragem por conglomerados, e utilizada a série de comandos "svy" do Stata ${ }^{16}$, considerando os setores censitários amostrados.

As variáveis foram descritas por meio de freqüências absolutas e relativas. As prevalências de hipertensão arterial de acordo com as variáveis independentes foram estimadas, testando-se a associação por meio do teste do qui-quadrado de Pearson. Aquelas que apresentaram significância estatística ao nível de significância de $20 \%(p<0,20)$ foram testadas na análise múltipla.

Para controle dos possíveis fatores de confundimento e por se tratar de um estudo transversal, aplicou-se a Regressão de Poisson $^{17}$, considerando-se para entrada das variáveis um modelo conceitual hierárquico adaptado de Sturmer et $\mathrm{al}^{18}$. Na abordagem hierarquizada, as variáveis de níveis mais distais são mantidas no modelo para ajuste das variáveis mais proximais ao desfecho, controlando o efeito das mesmas. Foram apresentadas, ao final, as Razões de Prevalência brutas e ajustadas, com respectivos intervalos de confiança de $95 \%$. 


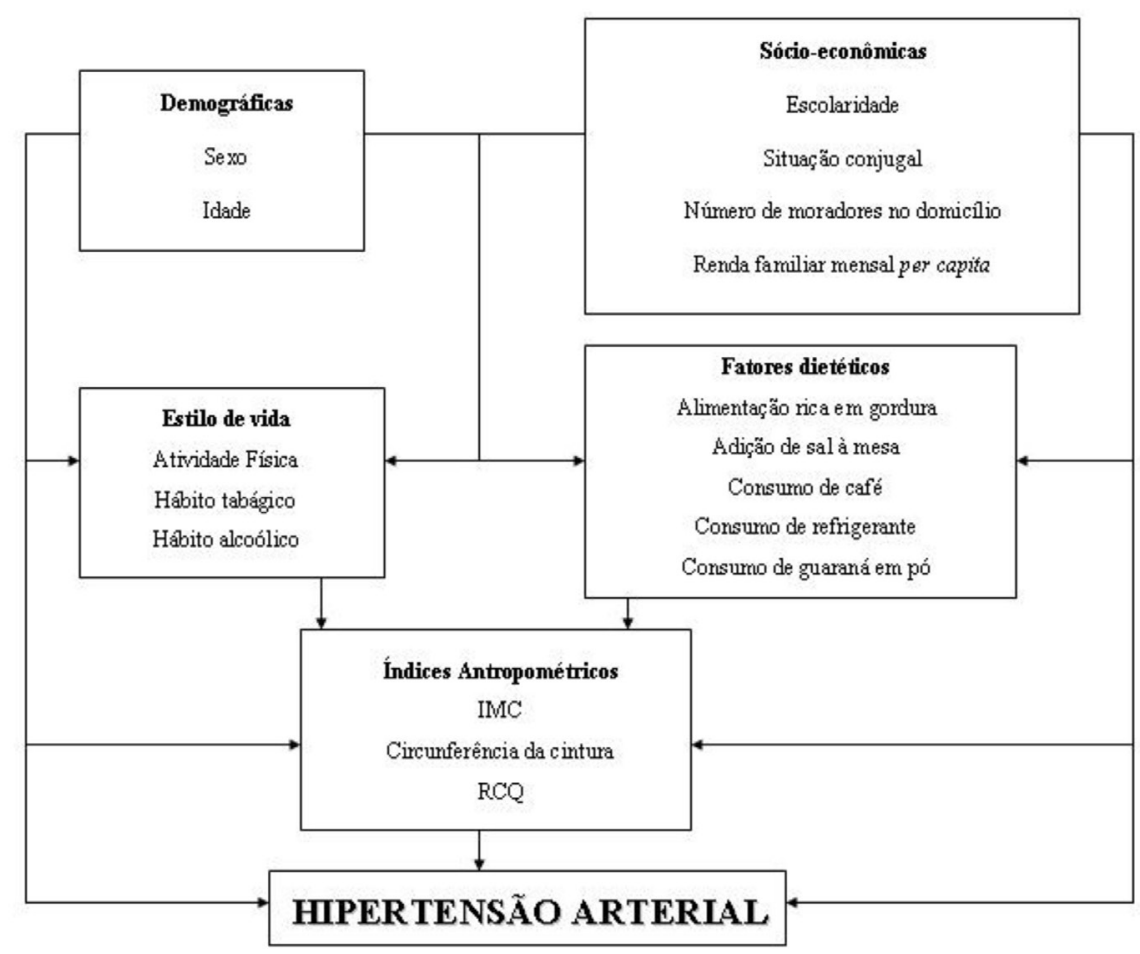

Figura 1 - Modelo conceitual hierárquico explicativo da hipertensão arterial, adaptado de Stumer et al (2006).

Figure 1 - Explanatory hierarchical concept model of blood pressure, adapted by Stumer et al (2006).

O projeto foi aprovado pelo Comitê de Ética em Pesquisa do Hospital Universitário Julio Müller, conforme a Resolução 196/96 do Conselho Nacional de Saúde.

\section{Resultados}

Foram avaliados 1.003 indivíduos residentes no município de Nobres -MT, sendo a maioria mulheres $(52,3 \%)$. Identificou-se 302 hipertensos $(30,1 \%)$, com prevalência maior no sexo feminino $(31,1 \%)$, porém sem diferença estatisticamente significativa entre os sexos $(\mathrm{p}=0,48)$. Indivíduos com mais idade, que não freqüentaram a escola $(60,6 \%)$, sem companheiros $(32,7 \%)$, residentes em domicílios com 1 a 3 moradores $(35,5 \%)$ e com renda familiar per capita igual ou superior a três salários mínimos $(32,0 \%)$ apresentaram maiores prevalências de hipertensão arterial (Tabela 1).

Quanto aos hábitos de vida e variáveis antropométricas, maiores prevalências de hipertensão foram observadas dentre os sedentários $(36,4 \%)$, ex-fumantes $(38,2 \%)$, não consumidores de bebidas alcoólicas (35,8\%), com índice de massa corporal igual ou superior a $25 \mathrm{~kg} / \mathrm{m}^{2}(41,9 \%)$, circunferência da cintura muito aumentada $(49,4 \%)$ e razão cintura-quadril de risco $(39,7 \%)$ (Tabela 2). Quanto aos hábitos alimentares, destacou-se a elevada prevalência de hipertensão dentre os consumidores de guaraná em pó (49,7\%).

Na análise univariada observou-se associação estatisticamente significativa entre a hipertensão arterial e a faixa etária, escolaridade, número de moradores no domicílio, prática de atividade física, hábito alcoólico, índice de massa corporal, circunferência da cintura, razão cintura-quadril e consumo de guaraná em pó.

Ajustando-se para possíveis fatores de confundimento e aplicando-se técnicas de 
Tabela 1 - Prevalência de hipertensão arterial segundo variáveis biológicas, socioeconômicas e demográficas na amostra analisada em Nobres-MT, 2006.

Table 1 - Prevalence of arterial hypertension per biological, socio-economic and demographic variable in the sample analyzed in Nobres-MT, 2006.

\begin{tabular}{|c|c|c|c|c|}
\hline Variável & $\mathrm{N}$ & Hipertensos & Prevalência (\%) & $\mathrm{p}^{*}$ \\
\hline \multicolumn{5}{|l|}{ Gênero } \\
\hline Feminino & 488 & 152 & 31,1 & 0,485 \\
\hline Masculino & 515 & 150 & 29,1 & \\
\hline \multicolumn{5}{|l|}{ Faixa etária } \\
\hline 18 a 29 anos & 261 & 20 & 7,7 & $<0,001$ \\
\hline 30 a 39 anos & 242 & 39 & 16,1 & \\
\hline 40 a 49 anos & 187 & 58 & 31,0 & \\
\hline 50 a 59 anos & 133 & 63 & 47,4 & \\
\hline 60 a 69 anos & 99 & 63 & 63,6 & \\
\hline 70 anos ou mais & 81 & 59 & 72,8 & \\
\hline \multicolumn{5}{|l|}{ Escolaridade ${ }^{\#}$} \\
\hline$\geq 8$ anos de estudo & 348 & 67 & 19,3 & $<0,001$ \\
\hline$<8$ anos de estudo & 497 & 140 & 28,2 & \\
\hline Não freqüentou escola & 109 & 66 & 60,6 & \\
\hline \multicolumn{5}{|l|}{ Situação conjugal } \\
\hline Sem companheiro & 379 & 124 & 32,7 & 0,161 \\
\hline Com companheiro & 624 & 178 & 28,5 & \\
\hline \multicolumn{5}{|c|}{ Número de moradores no domicílio } \\
\hline 1 a 3 & 448 & 159 & 35,5 & 0,004 \\
\hline 4 a 7 & 525 & 135 & 25,7 & \\
\hline 8 ou mais & 30 & 8 & 26,7 & \\
\hline \multicolumn{5}{|l|}{ Renda familiar per capita ${ }^{+}$} \\
\hline$\geq 3$ salários mínimos & 413 & 132 & 32,0 & 0,301 \\
\hline$<3$ salários mínimos & 588 & 170 & 28,9 & \\
\hline
\end{tabular}

Total de indivíduos $=1003=$ total number of people

*Referente ao teste do qui-quadrado de Pearson; ${ }^{*}$ Refers to Person's chi-square test

"49 pesquisados não responderam; \# 49 respondents did not answer

+2 pesquisados não responderam; $\uparrow 2$ respondents did not answer

análise múltipla, mantiveram-se significativamente associadas à hipertensão arterial a faixa etária e o índice de massa corporal. Indivíduos com 50 a 59 anos, 60 a 69 anos e 70 anos ou mais apresentaram prevalências cerca de seis, oito e nove vezes maiores que os aqueles com idade de 18 a 29 anos. Além disso, verificou-se que a prevalência de hipertensão arterial dentre os entrevistados com índice de massa corporal igual ou superior a $25 \mathrm{~kg} / \mathrm{m}^{2}$ foi $52 \%$ maior quando comparados aquele com IMC $<25 \mathrm{~kg} / \mathrm{m}^{2}$.

\section{Discussão}

O delineamento transversal do estudo não permite definir associações causais entre hipertensão e as características epidemiológicas estudadas, uma vez que, se tratando de um corte no tempo, os possíveis determinantes e os desfechos são vistos em um mesmo momento, impossibilitando identificar se a associação apresenta o critério de temporalidade. Apesar disso, levantar fatores associados à hipertensão arterial em 
Tabela 2 - Prevalência da hipertensão arterial segundo características de estilo de vida e características antropométricas dos 1.003 indivíduos estudados em Nobres-MT, 2006.

Table 2 - Prevalence of arterial hypertension according to lifestyle and anthropometric features of 1,003 people studied in Nobres-MT, 2006.

\begin{tabular}{|c|c|c|c|c|}
\hline Variável & $\mathrm{N}$ & Hipertensos & Prevalência (\%) & $\mathrm{p}^{*}$ \\
\hline \multicolumn{5}{|l|}{ Atividade física } \\
\hline Ativo & 301 & 77 & 25,6 & 0,012 \\
\hline Irregularmente ativo A & 272 & 72 & 26,5 & \\
\hline Irregularmente ativo B & 202 & 70 & 34,7 & \\
\hline Sedentário & 228 & 83 & 36,4 & \\
\hline \multicolumn{5}{|l|}{ Hábito tabágico } \\
\hline Não fumante & 677 & 201 & 29,7 & 0,201 \\
\hline Fumante & 237 & 67 & 28,3 & \\
\hline Ex-fumante & 89 & 34 & 38,2 & \\
\hline \multicolumn{5}{|l|}{ Hábito alcoólico } \\
\hline Não & 575 & 206 & 35,8 & $<0,001$ \\
\hline Sim & 428 & 96 & 22,4 & \\
\hline \multicolumn{5}{|l|}{ Índice de Massa Corporal } \\
\hline$<25 \mathrm{~kg} / \mathrm{m}^{2}$ & 480 & 83 & 17,3 & $<0,001$ \\
\hline$\geq 25 \mathrm{~kg} / \mathrm{m}^{2}$ & 523 & 219 & 41,9 & \\
\hline \multicolumn{5}{|l|}{ Circunferência da cintura } \\
\hline Normal & 440 & 81 & 18,4 & $<0,001$ \\
\hline Aumentada & 251 & 67 & 26,7 & \\
\hline Muito aumentada & 312 & 154 & 49,4 & \\
\hline \multicolumn{5}{|l|}{ Razão cintura-quadril } \\
\hline Normal & 613 & 147 & 24,0 & $<0,001$ \\
\hline Risco & 390 & 155 & 39,7 & \\
\hline
\end{tabular}

Total de 1.003 indivíduos; Referente ao teste do qui-quadrado de Pearson.

Total 1,003 people; Refers to Person's chi-square test

um município de pequeno porte contribui substancialmente para a implementação de políticas públicas de prevenção e controle, além de ampliar os conhecimentos existentes sobre os determinantes da doença.

Este estudo não apresenta perdas, considerando a estratégia de substituição adotada. Além disso, houve baixo percentual de recusas, o que pode ser explicado pela divulgação da realização do estudo no município, por meios de comunicação locais, antes e durante a pesquisa, e pela decisiva participação dos agentes comunitários de saúde. Ressalta-se que o município possui Programa de Agentes Comunitários de Saúde com cobertura de $100 \%$ dos domicílios da área urbana de Nobres.

Este estudo encontrou uma prevalência de $30,1 \%$ de HAS na população de 18 a 90 anos de idade, sem diferença significativa em relação ao gênero. Vale ressaltar que valores próximos de prevalência foram registrados em alguns estudos de base populacional, sob o mesmo ponto de corte $\geq 140 / 90 \mathrm{mmHg}$. Em Catanduva-SP, Freitas et al. ${ }^{19}$, avaliando 77.598 indivíduos na faixa etária maior de 18 anos, considerando o mesmo ponte de corte PAS $\geq 140$ e / ou PAD $\geq 90 \mathrm{mmHg}$, encontraram prevalência de hipertensão de $31,5 \%$, maior nos homens $(33,9 \%)$ do que nas mulheres $(29,9 \%)$. Lessa et al. ${ }^{20}$, em estudo realizado em Salvador-BA, 
Tabela 3 - Prevalência da hipertensão arterial segundo hábitos nutricionais da amostra analisada em Nobres-MT, 2006. Table 3 - Prevalence of arterial hypertension according to nutritional habits of the sample analyzed in Nobres-MT, 2006.

\begin{tabular}{|c|c|c|c|c|}
\hline Variável & $\mathrm{N}$ & Hipertensos & Prevalência (\%) & $p^{*}$ \\
\hline \multicolumn{5}{|c|}{ Alimentação rica em gordura } \\
\hline Não & 607 & 187 & 30,8 & 0,551 \\
\hline Sim & 396 & 115 & 29,0 & \\
\hline \multicolumn{5}{|c|}{ Adição de sal à mesa } \\
\hline Não & 927 & 280 & 30,2 & 0,818 \\
\hline Sim & 76 & 22 & 28,9 & \\
\hline \multicolumn{5}{|c|}{ Consumo de café } \\
\hline Não & 168 & 54 & 32,1 & 0,529 \\
\hline Sim & 835 & 248 & 29,7 & \\
\hline \multicolumn{5}{|c|}{ Consumo de refrigerante } \\
\hline Não & 109 & 40 & 36,7 & 0,112 \\
\hline Sim & 894 & 262 & 29,3 & \\
\hline \multicolumn{5}{|c|}{ Consumo de guaraná em pó\# } \\
\hline Não & 849 & 227 & 26,7 & $<0,001$ \\
\hline Sim & 151 & 75 & 49,7 & \\
\hline
\end{tabular}

Total de 1.003 indivíduos = total number of people

"Referente ao qui-quadrado de Pearson; * Refers to Person's chi-square test

"3 indivíduos não responderam; \# 3 peple did not answer

Tabela 4 - Regressão de Poisson com modelo conceitual hierárquico para os fatores associados à hipertensão em amostra de indivíduos de Nobres-MT, 2006.

Table 4 - Poisson's regression with a hierarchical concept model for factors associated to hypertension in a sample of people from Nobres-MT, 2006.

\begin{tabular}{lccc}
\hline Variáveis & $\mathrm{RP}_{\text {bruta }}$ & $\mathrm{RP}_{\text {ajustada }}$ & $\mathrm{IC95 \%}$ \\
\hline Faixa etária* & & & \\
$\quad 18$ a 29 anos & 1,00 & 1,00 & - \\
$\quad 20$ a 39 anos & 2,10 & $\mathbf{2 , 0 9}$ & $\mathbf{1 , 2 2 - 3 , 5 9}$ \\
40 a 49 anos & 4,04 & $\mathbf{4 , 0 0}$ & $\mathbf{2 , 4 0 - 6 , 6 7}$ \\
50 a 59 anos & 6,18 & $\mathbf{6 , 0 8}$ & $\mathbf{3 , 6 6 - 1 0 , 1 0}$ \\
$\quad 60$ a 69 anos & 8,30 & $\mathbf{7 , 9 6}$ & $\mathbf{4 , 7 7 - 1 3 , 3 1}$ \\
$\quad 70$ anos ou mais & 9,50 & $\mathbf{8 , 8 8}$ & $\mathbf{5 , 2 6 - 1 4 , 9 8}$ \\
Escolaridade (anos de estudo)* & & & - \\
$\quad \geq 8$ anos & 1,00 & 1,00 & $0,71-1,20$ \\
$\quad<8$ anos & 1,16 & 0,92 & $0,83-1,63$ \\
$\quad$ Não freqüentou a escola & 2,50 & 1,16 & - \\
Consumo de guaraná em pó** & & & $0,97-1,35$ \\
$\quad$ Não & 1,00 & - & - \\
$\quad$ Sim & 1,86 & 1,14 & $\mathbf{1 , 1 1 - 2 , 0 9}$ \\
Índice de Massa Corporal*** & & & $1,00-1,03$ \\
$\quad<25 \mathrm{~kg} / \mathrm{m}^{2}$ & 1,00 & 1,00 & $\mathbf{1 , 5 2}$ \\
$\quad \geq 25 \mathrm{~kg} / \mathrm{m}^{2}$ & 2,42 & 1,01 & \\
Circunferência da cintura*** & 1,65 & &
\end{tabular}

Razão de Prevalência (RP); Prevalence ratio

*Variáveis que ingressaram no primeiro nível; *Varibales included in the first level

**Variável que ingressou no segundo nível; ** Varibales included in the second level

*** Variáveis que ingressaram no terceiro nível; *** Varibales included in the third level 
com 1.439 adultos na faixa etária de maiores de 20 anos, considerando o mesmo ponto de corte, encontraram uma prevalência de $29,9 \%$, sendo maior nas mulheres $(31,7 \%$ vs. 27,4\%). Já em São Luis do Maranhão-MA, em 2008, avaliando-se 835 indivíduos maiores de 18 anos, a prevalência de hipertensão foi de $27,4 \%$, no sexo masculino $(32,1 \%)$ e no feminino $(24,2 \%)^{21}$.

Quanto à idade, observou-se que a prevalência de HAS aumentou progressivamente. A prevalência em Nobres foi de $63,6 \%$ em indivíduos de 60 a 69 anos e 72,8\% em indivíduos de 70 anos ou mais, enquanto em São Luis do Maranhão ${ }^{21}$ a prevalência foi de $70,7 \%$ acima dos 60 anos.

Quanto à escolaridade, verificou-se que os analfabetos apresentaram duas vezes mais hipertensão $(60,6 \%)$ quando comparados àqueles com oito ou menos anos de escola $(28,2)$. Em Goiânia-GO, Jardim et al. ${ }^{22}$, assim como em Nobres, a escolaridade apresentou associação inversa com a HA, com prevalência de $47,5 \%$ entre aqueles com menos anos de estudo e $28,0 \%$ com aqueles com mais de nove anos de estudo. Em São Luis do Maranhão, indivíduos com menos de 4 anos de estudo e mais de doze anos de estudos apresentaram maiores prevalências, $39,2 \%$ e $39,1 \%$ de $\mathrm{HA}^{21}$.

Não se observou associação positiva estatisticamente significativa entre a hipertensão arterial e o hábito de adicionar sal a alimentos prontos para consumo, sendo a prevalência de HA de 28,9\% e 30,2\% dentre os que adicionavam e não adicionavam, respectivamente. Tal resultado se mostrou consistente com o estudo de Freitas et al. (26,49\% vs. 25,44\%) ${ }^{19}$ em Catanduva-SP. Em Pelotas-RS, Dias da Costa et al. ${ }^{23}$, avaliando 1968 indivíduos de 20 a 60 anos e considerando hipertensos aqueles com PAS $\geq 160 \mathrm{e} /$ ou $\mathrm{PAD} \geq 95 \mathrm{mmHg}$, encontraram prevalência de HA de 13,6\% nos indivíduos que relataram adicionar sal na alimentação, e de $24,9 \%$ nos indivíduos que relataram não adicionar, diferindo do encontrado em Nobres-MT.

Quanto ao tabagismo, observou-se maior prevalência de hipertensão entre os ex-fumantes $(38,2 \%)$, quando comparados aos não fumantes $(29,7 \%)$, porém sem diferença estatisticamente significativa. Nesse estudo, indivíduos que referiram não fazer uso de bebidas alcoólicas apresentaram maior prevalência de HA do que aqueles que não as consumiram $(35,8 \%$ vs. $28,4 \%$; $\mathrm{p}<0,001)$. Este achado é controverso sob o ponto de vista epidemiológico, considerando que, neste caso, o álcool conferiria proteção em relação à hipertensão. Em Catanduva, Freitas et al. ${ }^{19}$ encontraram prevalência de hipertensão de 34,8\% entre os que relataram consumir mais de $30 \mathrm{ml}$ de etanol por dia $(\mathrm{p}=0,05)$. Ressalta-se a possibilidade de causalidade reversa, típica nos estudos transversais, além de que, neste estudo, não foi possível quantificar a ingestão de álcool, assim como do uso de cigarro.

Destaque-se a inédita e curiosa associação entre hipertensão e a ingestão de guaraná ralado, sob a forma de bebida com pequena quantidade de água e açúcar, merecendo novos estudos face à ampla difusão enquanto hábito regional de Nobres e Mato Grosso. Tal associação não se manteve significativa após ajuste em análise múltipla, possivelmente porque o consumo de guaraná ralado parece mais comum entre os indivíduos com maior idade, que apresentam substancialmente maiores prevalências de hipertensão quando comparados aos mais jovens.

$\mathrm{Na}$ analise ajustada permaneceram associados à HA a faixa etária, ou seja, sendo encontradas maiores prevalências com o avançar da idade, e o Índice de Massa Corporal $\geq 25 \mathrm{Kg} / \mathrm{m}^{2}$. Reafirma-se a necessidade de ações em nível populacional e individual direcionadas ao controle de peso corporal, principalmente em indivíduos com idade igual ou superior a 30 anos, o que, possivelmente, impactaria de forma importante sobre a prevalência de HA.

Este estudo revelou associações positivas entre hipertensão e fatores de risco bem conhecidos, caracterizado por indivíduos de idade mais elevada, com sobrepeso ou obesidade. Também, fortaleceu o conceito de que a prevalência da hipertensão arterial é alta em todo o país, independente da região e mesmo do tamanho das cidades. 


\section{Referências}

1. Ministério da Saúde. Sistema de Informações de Mortalidade (SIM). DATASUS. Óbitos causados por doenças do aparelho circulatório, 2002.

2. Lotufo PA. Mortalidade precoce por doenças do coração no Brasil. Comparação com outros países. Arq Bras Cardiol 1998; 70(5):321-325

3. Benetos A, Thomas F, Bean K, Gautier S, Smulyan J, Guize L. Prognostic value of systolic and diastolic blood pressure in treated hypertensive men. Arc Intern Med 2002; 162(5): 577-81.

4. Trindade IS, Heineck G, Machado JR, Ayzemberg H, Formighieri M, Crestani M, Gusso J. Prevalência da Hipertensão Arterial Sistêmica na População Urbana de Passo Fundo (RS). Arq Bras Cardiol 1998; 71(2): 127-30.

5. Gus I, Harzheim E, Zaslasvky C, Medina C, Gus M. Prevalência, reconhecimento e controle da hipertensão arterial sistêmica no Estado do Rio Grande do Sul. Arq Bras Cardiol 2004; 83(5): 424-428.

6. Sociedade Brasileira de Cardiologia. Sociedade Brasileira de Hipertensão. Sociedade Brasileira de Nefrologia. V Diretrizes Brasileiras de Hipertensão Arterial. São Paulo. Hipertensão, 2006; 9(4): 126-156.

7. IBGE - Instituto Brasileiro de Geografia e Estatística. Censo Demográfico 2000: Características gerais da população - resultados da amostra. Rio de Janeiro; 2000.

8. Ribeiro AB. Atualização em Hipertensão Arterial.SãoPaulo. Ed. Atheneu, 1996.

9. O'Brien E, Mee F, Atkins N, Thomas M. Evaluation of three devices for self-measurement of blood pressure according to the revised British Hypertension Society Protocol: the Omron Hem-705Cp, Philips HP 5332, and Nissei DS-175.

10. Organización Panamericana de la Salud. Protocolo y directrices: Conjunto de Ações para la reducción multifactorial de enfermedades no transmisibles (9 CARMEN/ CINDI). OPAS; 1997.

11. Matsudo SM, Matsudo VR, Araújo T, Andrade D, Andrade E, Oliveira L, Braggion G. Nível de atividade física da população do Estado de São Paulo: análise de acordo com o gênero, idade, nível socioeconômico, distribuição geográfica e de conhecimento. Rev Bras Ciên e Mov 2002; 10(4):41-50.

12. Lohman TG, Roger AF, Martorel R. Anthropometric standardization reference manual. Illiois: Human kinetics Books, 1988.

13. Callaway CW, Chumlea WC, Bouchard C, himes JH, Lohman TG, Martin AD et al. Circunference. In: Lohman TG, Roche AF, Martorell R. Anthropometric Standardization Reference Manual. Champaign, Illinois: Human Kinetics Books, 1988. p. 39-54.
14. Brasil. Ministério da Saúde. Vigilância alimentar e nutricional - Sisvan: orientações básicas para a coleta, processamento, análise de dados e informação em serviços de saúde. Brasília: Ministério da Saúde; 2004. $120 \mathrm{p}$.

15. Decano AG, Arner TG, Sangan S, Sunki GG, Friedman R, Lantiga M, Zubieta JC, Sullivan KM, Smithe DC. Epi-Info 2000, um banco de dados e programa de estatística para profissionais de saúde pública para uso em Windows 95,98, NT e 2000. Centro para Controle e Prevenção de Doenças, Atlanta, Geórgia, EUA 2000.

16. StataCorp LP. Stata/IC 10,0 for Windows. Texas: College Station; 2007.

17. Barros AJ, Hirakata VN. Alternatives for logistic regression in cross-sectional studies: an empirical comparison of models that directly estimate the prevalence ratio. BMC medial research methodology 2003; 3: 21.

18. Sturmer G, Dias-da-Costa JS, Olinto MTA, Menezes AMB, Gigante DP, Macedo S. O Manejo não medicamentoso da hipertensão arterial sistêmica o Sul do Brasil. Cad Saúde Pública 2006; 11(8): 1727-37.

19. Freitas OC, Carvalho FR, Neves JM, Veludo PK, Parreira RS, Gonçalves RM, et al. Prevalência de hipertensão arterial sistêmica na população urbana de Catanduva, SP. Arq Bras cardio 2001, 77(1): 9-15.

20. Lessa I, Mahalhães L, Araujo MJ, de Almeida Filho N, Aquino E, Oliveira MM. Arterial hypertension in the adult population of Salvador- BA. Arq. Bras. Cardiol 206; 87 (6): $747-56$.

21. Barbosa JB, da Silva AAM, dos Santos AM, Monteiro Junior FC, Barbosa MM, Barbosa MM, et al. Prevalence of arterial hypertension and associated factors in adults in São Luís, state of Maranhão. Arq.Bras Cardiol 2008; 91 (4): 260-266).

22. Jardim PCV, Peixoto MR, Monego E Moreira H, Vitorino PVO, Souza WSBS, Scala LCN. Hipertensão Arterial e Alguns Fatores de Risco em uma Capital Brasileira. Arq Bras Card 2007; 88(4): 452-57.

23. Dias da Costa JS, Barcellos FC, Sclowitz ML, Sclowitz IK, Castanheira M, Olinto MT, et al. Hypertension prevalence and its associated risk factors in adults: a population-based study in Pelotas. Arq.Bras.Cardiol 2007 88(1): 59-65.

Recebido em: 28/11/08 Versão final reapresentada em: 29/04/09 Aprovado em: 04/05/09 